\title{
Imaging of intrahepatic progression of hepatocellular carcinoma post transarterial chemoembolization. A long-term, prospective evaluation of contrast-enhanced ultrasonography (CEUS)
}

\author{
Hippocrates Moschouris ${ }^{1}$, Mariana Kalokairinou-Motogna' ${ }^{2}$, Spyros Vrakas, \\ Aggeliki Papadatou ${ }^{1}$, Eyaggelos Karagiannis ${ }^{1}$, Michail Kiltenis ${ }^{1}$, Konstantinos Kladis-Kalentzis ${ }^{1}$, \\ Kyriaki Marmaridou ${ }^{1}$, Nikolaos Papadogeorgopoulos ${ }^{1}$, Katerina Malagari ${ }^{4}$
}

${ }^{1}$ Department of Radiology, Tzaneion General Hospital, Piraeus, ${ }^{2}$ Private Practice, Athens, ${ }^{3}$ Department of Gastroenterology, Tzaneion General Hospital, Piraeus, ${ }^{4}$ Second Department of Radiology, University of Athens, Attikon Hospital, Chaidari, Athens, Greece

\begin{abstract}
Aims: To assess the diagnostic efficacy of contrast-enhanced ultrasonography (CEUS) in the context of intrahepatic progression (IHP) of hepatocellular carcinoma (HCC) after transarterial chemoembolization (TACE). Material and methods: Sixty HCC patients were prospectively included in the study. They were treated with transarterial chemoembolization (TACE) with doxorubicin-eluting microspheres (231 sessions). Imaging follow-up was performed 1 month after each session and at 3-6 month intervals after the last session of TACE and included CEUS and contrast-enhanced magnetic resonance (MR) imaging (reference modality). The diagnosis of IHP was based on mRECIST criteria and the respective findings of MR and CEUS were recorded, categorized and correlated. Results: A total of 441 CEUS studies were compared with the corresponding MR studies. During a follow-up period of 5-82 months (mean: 22 months), MR diagnosed 51 cases of IHP in 34/60 (56.6\%) patients. CEUS correctly diagnosed 12/14 (85.7\%) cases of IHP of target tumors, $2 / 5(40 \%)$ cases of IHP of non-target tumors, $13 / 18$ $(72.2 \%)$ cases of distal and 6/9 (66.6\%) cases of proximal new lesions, and 5/5 (100\%) cases of major vessel involvement. On a per-lesion basis, CEUS was significantly inferior to MR in the detection of new lesions $(\mathrm{p}=0.002)$. No false positive CEUS diagnoses of IHP were observed. 54\% of the diagnostic failures of CEUS were considered clinically significant. Conclusion: In the long term evaluation of HCC post TACE, CEUS appears to have limitations in the detection of IHP, which are more prominent in the case of new lesions and of progressive non-target tumors.
\end{abstract}

Keywords: hepatocellular carcinoma; intrahepatic progression; transarterial chemoembolization; contrast-enhanced ultrasonography; magnetic resonance imaging.

\section{Introduction}

Among its other applications in liver imaging, contrast-enhanced ultrasonography (CEUS) is being utilized with increasing frequency in the setting of peri- and postinterventional imaging of liver tumors [1]. By providing real-time, multiplanar imaging of tumoral enhance-

Received 10.12.2016 Accepted 24.02.2017

Med Ultrason

2017, Vol. 19, No 2, 134-142

Corresponding author: Mariana Kalokairinou-Motogna 42 Terpsitheas, 15341 Ag. Paraskevi, Athens, Greece

Phone: 00306934000743

E-mail: motogna@hotmail.com ment with adequate spatial resolution, CEUS has been proven highly efficient in the differentiation of the viable (enhancing) from the necrotic (non-enhancing) components of the treated tumors. This advantage is much more prominent in hypervascular tumors, such as hepatocellular carcinoma (HCC), and is utilized with satisfactory results for interventional guidance and for short-term response evaluation of $\mathrm{HCC}$ treated with ablative or embolisation procedures [2]. For diagnosis of residual viable tumor post ablation, the sensitivity of CEUS is $83.3-93.3 \%$ with a specificity of $98-100 \%$ [3]; the respective values for evaluation post transarterial chemoembolization (TACE) are $87-100 \%$ and $83.3-100 \%$ [4-7]. To further assess the clinical role of post-interventional 
CEUS and to incorporate CEUS into a standardized post-interventional approach of $\mathrm{HCC}$, two studies $[7,8]$ attempted to combine the widely accepted mRECIST system (modified Response Evaluation Criteria In Solid Tumors) [9] with post-TACE CEUS imaging of HCC. It was concluded that this combination is feasible and has not only a diagnostic, but also prognostic value. Notably, the two aforementioned studies and a few others $[10,11]$ were not confined in the evaluation of the target tumors; non-target tumors and the rest of the liver were also evaluated and potential limitations of CEUS were revealed, when CEUS was utilized for a global evaluation of the liver. We believe that these limitations may be clinically relevant and merit further investigation. In the long-term course of HCC patients post interventional treatment, disease progression involving non-target tumors, or (perhaps more importantly) new lesions, is common and may have an impact on prognosis and on treatment decisions $[12,13]$. In early-stage HCC treated with radiofrequency ablation (RFA), distal recurrences have been observed in $10.4 \%$ and $52.5 \%$ of patients at 1 and 3 years post treatment, respectively [14]. In intermediate-stage HCC treated with TACE, the rates of distal recurrences are also high (23-57\% in the 1 st year and $50-100 \%$ at 3 years post treatment) [13].

This study is based on our 8 years' experience with post interventional (particularly post-TACE) imaging of HCC with CEUS, along with a standard imaging modality. Focusing on the various types of intrahepatic progression of the disease, the respective CEUS findings are reviewed, categorized and correlated with those of the reference standard.

\section{Materials and methods}

\section{Patients}

This was a prospective study of HCC patients ineligible for surgery, who were treated with TACE in our department. The first patient was enrolled in the study on March 2008 and the last on April 2015. All patients were newly diagnosed with HCC, according to AASLD guidelines [15]. To ensure adequate follow-up time, we included patients who were studied with the appropriate imaging protocol for at least 5 months. We excluded patients with history of prior surgical or interventional, liver-directed treatments and patients with incomplete or technically inadequate imaging studies. Also excluded were patients with diffusely infiltrating HCCs, since these tumors were unsuitable for application of any standard system of response criteria.

For each patient, the follow-up period was considered to begin at the time of the first treatment and ended at the time of patient's death, or at the time of patient's last visit, or when a treatment other than TACE was undertaken.

Prior to treatment initiation, all patients had been informed in detail regarding the therapeutic and posttreatment imaging protocol and had provided written informed consent. The study was approved by the Institutional Review Board.

\section{TACE}

TACE with drug-eluting microspheres was applied to all the patients of this study. Approximately $30 \%$ of the interventions were performed with 100-300 $\mu \mathrm{m}$ DCBeads (Biocompatibles Ltd, Surrey, UK), while $50 \%$ of the interventions were performed with Hepasphere microspheres (Biosphere Medical, Paris, France), with dry state diameter of 50-100 $\mu \mathrm{m}$. For the remaining sessions, $75 \mu \mathrm{m}$ or $100 \mu \mathrm{m}$ Embozene-Tandem (CeloNovaBioSciences, CA, USA) was utilized. The microspheres had been preloaded with doxorubicin (Adriblastina, Pfizer Italia S.r.1., Nerviano, Milano, Italy) at a dose of 50-75mg drug/vial. Other technical aspects of TACE and inclusion criteria were similar to that described in previous studies [16]. The treatment protocol included at least 3 scheduled TACE-sessions (at 5-7 week intervals), unless complete response was achieved after the first, or second session. Further treatment decisions were made according to the relevant official technical recommendations [17].

\section{Reference imaging modality}

Imaging follow-up was performed 1 month after each session of TACE and at 3-6 month intervals after the last session. Contrast-enhanced magnetic resonance (MR) imaging served as the reference standard. Although more than one MR units were utilized, the following technical prerequisites had to be met: i) Imaging at field strengths of 1.5 or 3.0 Tesla; ii) Contiguous slices with a thickness equal to, or less than 5mm; iii) Axial and coronal, unenhanced T1 and T2-weighted images; iv) axial and coronal dynamic (gadolinium-enhanced) T1 sequences, depicting tumor vascularization in the arterial, portal and delayed phases. The extracellular paramagnetic agent gadopentatedimeglumine (Magnevist, Bayer HealthCare) at a dose of $0.2 \mathrm{~mL} / \mathrm{kg}$ was used for these sequences. v) Absence of significant artifacts. MR studies were stored in DICOM (Digital Imaging and Communications in Medicine) format in a personal computer and were reviewed with dedicated software. The radiologist who reviewed these studies had 20 years experience in cross sectional abdominal imaging (10 years in post-interventional imaging) and was blinded to the findings of CEUS.

\section{Sonographic imaging protocol}

The schedule of the sonographic follow-up was similar to that of standard imaging. Two ultrasonographic units (Philips HD11 XE, Philips Ultrasound, Andover, 
MA, USA, and General Electric Logiq E9, GE Healthcare, Milwaukee, WI, USA) with CEUS capability and with multifrequency (1-5 MHz or 2-5 MHz), curved array transducers were utilized.

Our standard post-interventional sonographic protocol included: 1) Unenhanced, B-mode and Color-Doppler ultrasonography (US) for the study of: i) target and non-target tumors (size, borders, echogenicity), ii) major vessels (portal, hepatic veins and intrahepatic portion of inferior vena cava-IVC) for patency and signs of tumor invasion, iii) rest of liver (for new lesions, complications, deterioration of cirrhosis); 2) CEUS study, which was accomplished in 2 phases, each of them requiring a separate injection of echo-enhancer. The first phase primarily included scanning of the target tumors, to visualize the full extent of their enhancing (viable) components and to evaluate their response, according to the current guidelines [18]. Major vessels and non-target or new lesions in the vicinity of the target tumors could also be imaged at this phase. At the delayed period of scanning (more than 120 seconds post $1^{\text {st }}$ injection), the rest of the liver could also be scanned to detect enhancement defects caused by the wash-out of potential new lesions. The second phase was focused on liver areas and on non-target and/or new lesions that were not completely studied during the first phase. Lesions that had been picked up as enhancement defects during the end of the first phase were scanned, to determine the degree of their arterial enhancement. All CEUS studies were performed with the currently commercially available in Europe, second generation ultrasound contrast agent (suspension of microbubbles of sulphur hexafluoride, [SonoVue, Bracco, Milan Italy]), which was injected as a bolus in a forearm vein, followed by a flush of normal saline. The dose of the echo-enhancer for each injection was $2.4 \mathrm{ml}$ (for scans performed in GE E9 machine) or $4.8 \mathrm{ml}$ (for Philips HD11). A dedicated, contrast specific, continuous scanning, low mechanical index (MI=0.08-0.14) algorithm was utilized and the liver was scanned for 240 seconds after each injection. There was a 7-10 minute interval between the two steps of the CEUS study in the same patient.

Images of the unenhanced US studies and full-length video acquisitions of the CEUS studies were digitally stored in the hard disk of the ultrasonographic units and were reviewed on the monitor of each unit. The target, non-target and new lesions were identified and measurements (maximum diameter of the entire lesion and of its enhancing part) were performed. The findings were correlated with those of previous studies, to diagnose IHP (the respective criteria will be discussed below).

Based on previous experience with factors adversely affecting the diagnostic efficacy of CEUS [19], we re- corded the following baseline features of the sonographic studies, in order to assess their effect on diagnostic performance of CEUS: 1) Distance of the studied lesion from the transducer (more or less than $10 \mathrm{~cm}$ ); 2) Enhancement pattern of the studied lesion (typical, with arterial hyperenhancement and portal or delayed hypoenhancement, versus any other); 3) Multiplicity of HCC at baseline (up to 3 focuses, versus more); 4) Degree of cirrhosis-related heterogeneous enhancement of liver parenchyma (a subjective visual assessment was performed to differentiate little or no heterogenicity from severe hererogenicity of liver parenchymal enhancement).

All CEUS examinations and subsequent analysis were performed by one radiologist (with 9 years experience in peri- and post-interventional CEUS), who was unaware of the corresponding MR findings.

\section{Other investigations}

Laboratory tests (blood, biochemical tests and serum alpha-fetoprotein, AFP levels) were obtained with a schedule similar to that of the imaging follow up. Additional imaging was also performed to address individual issues that occurred during follow up. For example, chest computed tomography was performed in patients with increasing levels of AFP in the absence of intraabdominal progression.

\section{Imaging diagnosis of intrahepatic progression and correlations}

For the purposes of this study, any manifestation of recurrent or progressive $\mathrm{HCC}$ confined in the liver was considered as "Intrahepatic Progression" (IHP). Imaging criteria for the diagnosis of IHP were largely based on mRECIST system [9], with a few amendments, to facilitate diagnosis in equivocal cases. The official guidelines for CEUS in the liver were also taken into account [18]. The same imaging criteria were used for the MR-and for the CEUS-diagnosis of IHP. The following patterns of IHP were defined:

- IHP of target tumors. This was defined according to mRECIST, as "an increase of at least $20 \%$ in the sum of the diameters of viable (enhancing) target lesions, taking as reference the smallest sum of the diameters of viable (enhancing) target lesions recorded since the treatment started" [9]. For patients with more than two measurable tumors at baseline, the two largest lesions were considered as "target tumors" and the rest were considered as non-target tumors. In clinical practice, some target-HCCs treated with TACE show atypical enhancing features, such as arterial iso- (instead of hyper-) enhancement, or hypoenhancement at all three phases of the study $[18,20]$. We decided not to exclude such tumors, provided that: i) they were well defined (and thus easily measurable), ii) their atypically enhancing components could be clearly differenti- 
ated from necrotic (non-enhancing) components and, iii) they had been definitely diagnosed as HCCs at baseline. In such tumors, the largest diameter of the atypically (instead of arterially) enhancing component was measured, to assess the potential progression of target tumors.

- IHP of non-target tumors. mRECIST defines this type of IHP as "an unequivocal progression of existing non-target lesions" [9]. We defined IHP as an increase of at least $20 \%$ in the diameter of at least one viable nontarget lesion, taking as reference the smallest diameter of this lesion since the treatment started.

- IHP in the form of new lesions. As per mRECIST guideline, newly appearing nodules were diagnosed as new lesions, when they demonstrated a typical vascular pattern on dynamic imaging (i.e hypervascularization in the arterial phase with washout in the portal venous or late venous phase), and a maximum diameter of at least $1 \mathrm{~cm}$. For newly appearing, atypically enhancing nodules $(\geq 1 \mathrm{~cm}$ in diameter) we adopted less strict criteria than mRECIST: such lesions were diagnosed as HCCs if, i) they showed an interval (3 months) growth of at least $5 \mathrm{~mm}$ on follow-up imaging, or, ii) they were associated with unequivocal increase of AFP, in the absence of other manifestations of intra- or extrahepatic progression. New lesions arising in the same liver segment as the target tu-

Table I. Demographic and clinical data of the studied patients at baseline.

\begin{tabular}{ll}
\hline Age (yrs) mean+/-SD & $66.9+/-9.4$ \\
Sex (M/F) & $48 / 12$ \\
Cirrhotic background & $58 / 60$ \\
$\quad$ HBV & 41 \\
$\quad$ HCV & 7 \\
$\quad$ Alcohol & 3 \\
Combined causes & 6 \\
Unknown & 1 \\
Child's classification (A/B) & $43 / 17$ \\
BCLC stage (A/B/C) & $5 / 42 / 13$ \\
Tumor number & \\
Solitary/Two/Three/Four or more & $16 / 15 / 13 / 16$ \\
Diameter of targets* (mm): & \\
Minimum/Maximum/Mean/Median & $31 / 252 / 81 / 70$ \\
* In cases with 2 target tumors, the sum of maximum diameters is \\
provided
\end{tabular}

mors were considered as "proximal" and were examined separately from those appearing in other liver segments ("distal" new lesions).

- IHP in the form of major vessel involvement. Events of neoplastic thrombosis of portal, or hepatic veins, or of the intrahepatic portion of inferior vena cava (IVC) were registered under this separate category.

In an individual patient, the MR or CEUS diagnosis of IHP at a specific time point and in any of the 5 aforementioned patterns was considered as a separate "case" of IHP. The number of lesions involved in each "case" of IHP was calculated. CEUS diagnosis of IHP was compared with MR on a "per case" and on a "per lesion" basis.

\section{Statistic analysis}

Descriptive statistics were calculated for quantitative and qualitative data. The normality of the distribution of numerical variables was assessed with the Shapiro-Wilk test. Differences in numerical variables between CEUS and MR were assessed with the t-test and theWilcoxon signedrank test. Factors which could potentially affect the performance of CEUS in diagnosis of IHP were evaluated with univariate and multivariate analysis. The time to IHP (cumulatively and separately for the main patterns) was calculated with the Kaplan-Meier method. Statistical significance was defined as a $p$ value of $<0.05$. Statistical analysis was performed with SPSS 19.0 (SPSS Inc., Chicago, IL).

\section{Results}

The study included 60 patients (age: $45-85$ years) with 99 target tumors and 63 non-target tumors at baseline (table I).

Fifty-four patients $(90 \%)$ had typically enhancing tumors at baseline. A total of 231 sessions of TACE (2-12 sessions per patient, median: 4 sessions per patient) were performed for the treatment of the patients of this study. The follow-up period ranged from 5 to 82 months (mean: 22 months, median: 15 months). A total of 441 CEUS studies (3-26 studies per patient, mean: 7, median: 5 studies per patient) and the same numbers of corresponding MR studies were performed during that period, for the evaluation of IHP.

Table II. Comparison between the reference modality (MR) and CEUS in the diagnosis of various patterns of IHP.

\begin{tabular}{llll}
\hline & Diagnosed by MR & Missed by CEUS & Underdiagnosed by CEUS \\
\hline Progression of target tumors & 14 & $2[2]$ & 0 \\
Progression of non-target tumors & 5 & $3[1]$ & 0 \\
New lesions: Proximal & 9 & $3[2]$ & 2 \\
New lesions: Distal & 18 & $5[2]$ & $6[2]$ \\
Major vessel involvement & 5 & 0 & 1 \\
Total & 51 & $13[7]$ & $9[2]$ \\
\hline
\end{tabular}

Figures represent numbers of cases. Figures in brackets represent numbers of clinically significant cases (see text). 
MR diagnosed a total of 51 cases of IHP in 34 of the $60(56.6 \%)$ patients of the study. Nine of the 34 patients experienced more than one case of IHP at the same, or at different phases of follow-up. CEUS diagnosed 38 of the 51 cases of IHP (overall sensitivity: $74.5 \%$ ). Regarding the different patterns of IHP, the following observations were made (table II):

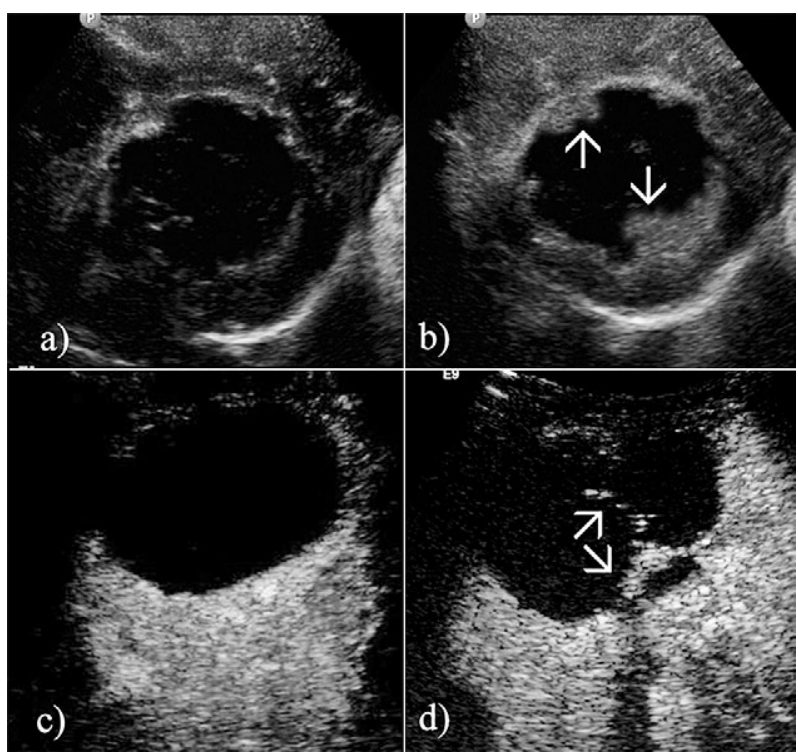

Fig 1. Successful CEUS diagnosis of local tumor recurrence (2 cases). In the first case, the image from a CEUS study (a), performed one month after the 3rd session of TACE, shows minimal residual enhancement at the periphery of the target tumor. Further treatment was postponed, because of impairment of liver function. Image from a follow-up CEUS study 4 months later (b), shows definite increase of the nodular enhancing tissue at tumor's periphery (arrows). In the second case, the image from a CEUS study (c), performed one month after the 3rd session of TACE, shows complete disappearance of tumoral enhancement. The image from a follow-up CEUS study 3months later (d), shows reappearance of band-like enhancing tissue (arrows). In both cases the total tumor size remained stable.
MR diagnosed 14 cases of IHP of target tumors (14 lesions, one lesion per case). In 4 lesions, recurrence of intratumoral enhancement occurred in previously completely necrotic targets. No significant change in total tumor size was observed in these cases. The remaining 10 lesions had shown partial or no response to TACE, and IHP appeared as a $20-39 \%$ enlargement of preexisting enhancing components (6/10 lesions), or as a $20-27 \%$ global tumoral growth (4/10 lesions). CEUS correctly diagnosed 12/14 cases of IHP of target tumors (sensitivity: $85.7 \%$ ) (fig 1).

The morphology and size of CEUS findings in these cases were similar to those of MR. CEUS missed 2 of the 4 cases of reappearance of intratumoral enhancement in previously completely necrotic tumors. One of these lesions had a subphrenic location, $12.5 \mathrm{~cm}$ from the skin surface, and showed a $4 \mathrm{~mm}$ peripheral nodular recurrence on MR. In the other, the peripherally located recurrent tumor $(3.8 \times 10 \mathrm{~mm})$ was probably obscured by acoustic shadowing caused by a fibrous capsule at the tumor's periphery (fig 2).

MR diagnosed 5 cases of IHP of non-target tumors, in the form of global enlargement (20-26\%) of the involved lesions. CEUS failed to diagnose IHP in 3 of these cases (3 lesions), thus the sensitivity of CEUS for this pattern was $40 \%$. One of those lesions was not easily discernible from the surrounding parenchyma in both US and CEUS. The other two failed diagnoses occurred in livers with numerous non-target tumors; adequate depiction of each of them proved impossible.

MR diagnosed a total of 27 cases of IHP in the form of new lesions ( 9 cases of proximal and 18 cases of distal new lesions). Corresponding CEUS studies failed to diagnose 8 of these 27 cases. Therefore, on a per case basis, the sensitivity of CEUS in detection of new lesions was $70.3 \%$. Of the remaining 19 cases, CEUS underestimated the number of new lesions in 8 cases. On a per-lesion basis,
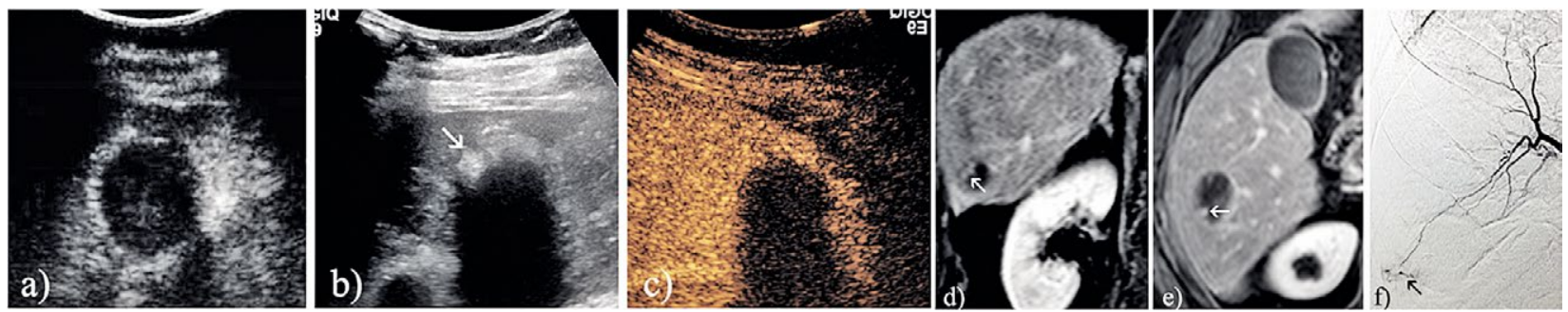

Fig 2. CEUS failure in diagnosis of local tumor recurrence. The image from a CEUS study (a), 6 months after the second session of TACE, shows complete lack of enhancement of the target tumor, indicative of complete necrosis. This was confirmed by MR (not shown). The image from unenhanced US study (b), 3 months later, shows an echogenic capsule at the periphery of the lesion (arrow) with associated dense acoustic shadowing. The latter obscures a large part of the tumor at the corresponding CEUS image (c). Corresponding T1 weighted, contrast-enhanced coronal (d), and axial (e), MR images, show nodular enhancement at the periphery of the tumor (arrows), consistent with local tumor recurrence that was missed by CEUS. Angiographic image (f) prior to embolisation for the treatment of this recurrence shows also the tumor stain (arrow). 
CEUS detected significantly fewer new lesions than MR $(\mathrm{p}=0.002)$ : MR detected a total of 139 new lesions (mean: $5.1+/-4.3$ new lesions per case), while CEUS detected a total of 85 new lesions (mean: 3.1+/- 2.8 new lesions per case). Thus, on a per lesion basis, the sensitivity of CEUS in detecting new intrahepatic recurrences was $61.1 \%$. The size (maximum diameter) of the new lesions detected by MR (mean: $16.3+/-5.2 \mathrm{~mm}$ ) did not differ from the size of the new lesions detected by CEUS (mean: $16.5+/-5.4 \mathrm{~mm}$, $\mathrm{p}=0.93)$. Of note, approximately two thirds $(55 / 85,64.7 \%)$ of the new lesions diagnosed by CEUS had been initially detected at the unenhanced US study that preceded CEUS. The remaining 30 lesions had not been detected by unenhanced US, but became evident by means of wash-out during the first step of the CEUS study (fig 3 ).

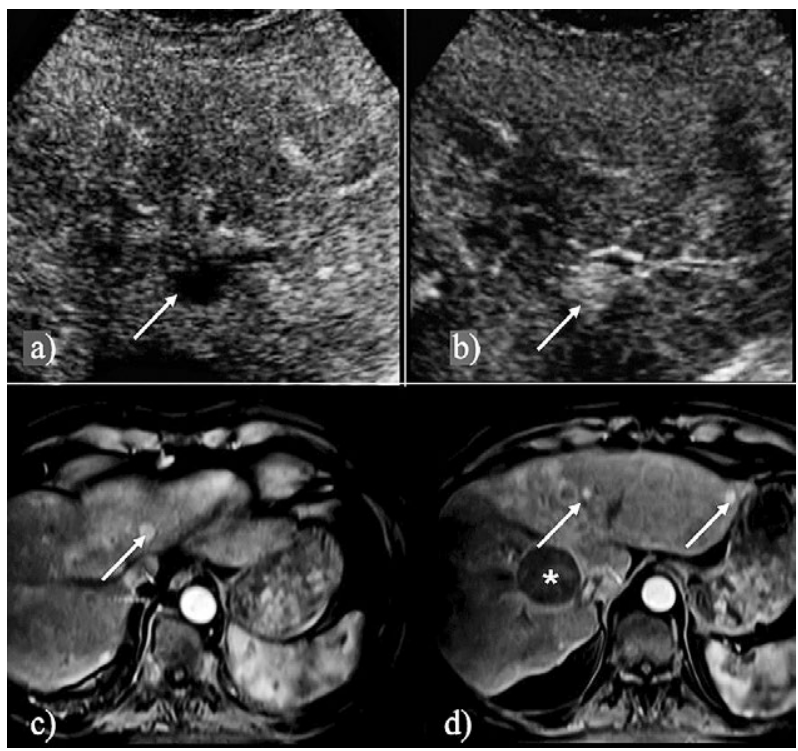

Fig 3. Detection of new lesion by CEUS. The image from the delayed phase of the first part of CEUS examination (a), shows a new hypoenhancing lesion (arrow) at the left liver lobe. The image from the early phase of the second part (second injection) of CEUS (b), shows that the lesion has intense arterial enhancement (arrow). Corresponding axial T1-weighted, contrast enhanced-arterial phase-MR image (c), confirms the distal recurrence (arrow). Axial T1-weighted, contrast enhanced-arterial phase-MR image at lower level (d), shows additional arterially enhancing focuses (arrows) some of which were missed by CEUS. The necrotic target tumor is also depicted (asterisk).
Primary detection of new lesions solely on the basis of their arterial hyperenhancement proved impossible with our protocol.

Finally, MR diagnosed 5 cases of major vessel involvement (4 cases of malignant portal vein thrombosis and one case of combined right hepatic and intrahepatic IVC malignant thrombosis). CEUS also diagnosed the same 5 cases, although it underdiagnosed the last one, by missing the right hepatic vein component. All of the cases of major vessel involvement were associated with local progression of adjacent target tumors.

On the basis of their impact on classification of response (per mRECIST) and on treatment decisions, 7 out of $13(54 \%)$ missed CEUS diagnoses of IHP were considered clinically significant. Two of the cases of underestimation of IHP by CEUS were also considered clinically relevant since MR detected more new lesions in these cases; it was decided to treat them with TACE instead of ablation.

IHP of target tumors tended to occur earlier than IHP caused by new lesions (11.6+/-6.2 months post first treatment versus $18.5+/-14.3$ months post first treatment, $\mathrm{p}=0.043)$. Only $3 / 51(5.9 \%)$ cases of IHP were observed during the follow-up period of the first 3 scheduled session of TACE. CEUS correctly diagnosed these 3 cases, although it underestimated the number of new lesions in one of them. More details regarding the temporal distribution of the various forms of IHP are provided in table III and figure 4.

No false-positive CEUS diagnoses of any type of IHP were observed.
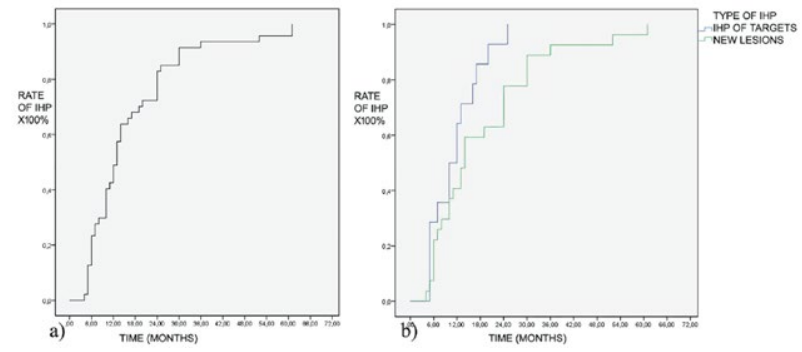

Fig 4. Kaplan-Meier plots for the rates of IHP cumulatively for all the patterns (a), and separately for the two patterns of IHP with the largest numbers of cases (b).

Table III. Temporal distribution of the cases of IHP and of the respective CEUS failures

\begin{tabular}{|c|c|c|c|c|c|c|c|}
\hline & $\begin{array}{l}\text { During follow-up of the first } \\
3 \text { scheduled treatments }\end{array}$ & $\begin{array}{l}<6 \\
\text { mo }\end{array}$ & $\begin{array}{l}\text { 6-12 } \\
\text { mo }\end{array}$ & $\begin{array}{l}\text { 13-18 } \\
\text { mo }\end{array}$ & $\begin{array}{l}19-24 \\
\text { mo }\end{array}$ & $\begin{array}{l}\text { 25-30 } \\
\text { mo }\end{array}$ & $\begin{array}{l}>30 \\
\text { mo }\end{array}$ \\
\hline Progression of target tumors & 2 & 4 & $5[1]$ & 3 & 1 & $1[1]$ & 0 \\
\hline Progression of non-target tumors & 0 & 0 & $3[2]$ & $1[1]$ & 1 & 0 & 0 \\
\hline New lesions & 1 & $2[1]$ & $10[3]$ & $5[1]$ & $5[1]$ & $3[2]$ & 2 \\
\hline Major vessel involvement & 0 & 0 & 3 & 2 & 0 & 0 & 0 \\
\hline
\end{tabular}

Figures represent numbers of cases. Figures in brackets represent numbers of cases missed by CEUS 
On univariate analysis, lesion multiplicity $(\mathrm{p}=0.001)$, atypical tumoral enhancement $(\mathrm{p}=0.002)$ and deep location of the studied tumors $(\mathrm{p}=0.05)$, were found as significant predictors of a failed CEUS diagnosis of IHP. The first two of the aforementioned factors retained their significance on multivariate analysis.

\section{Discussions}

Several studies [4-6] have evaluated CEUS as an alternative modality for the monitoring of $\mathrm{HCC}$ after TACE. However, the majority of them are based on short-term, focused evaluation of the target tumors after a single treatment session; data regarding the role (if any) of CEUS in the long-term follow-up and in detection of IHP are still limited. Moreover, the clinical relevance of the failed diagnoses of CEUS has not been previously evaluated. Through the longitudinal study of our patients with serial CEUS examinations and for adequate followup time, we observed several CEUS patterns of IHP and we compared CEUS findings with those of the reference standard. We used MRI (instead of CT) as reference method for two reasons: First, according to the protocol applied to all HCC-patients treated with TACE in our institution, MR is the standard pre- and post-interventional imaging modality. Second, MR is at least equal, if not superior to CT for the study of HCC post TACE. In the context of conventional (lipiodol) TACE, MR is clearly superior to $\mathrm{CT}$, since MR is free from artifacts which are caused by lipiodol and which interfere with detection of viable (enhancing) tissue by CT. Regarding the evaluation of TACE with drug-eluting microspheres, the two modalities show similar diagnostic performance [21]. Moreover, according to a recent metaanalysis, MR was significantly more sensitive than CT in diagnosing HCC in patients with chronic liver disease $(p=0.0023)$ [22]. Limitations of MR include longer scanning times and lower availability compared to CT, as well as patients' intolerance and contraindications to exposure to the magnetic field.

The observed CEUS patterns of IHP were similar to the patterns that have already been described with $\mathrm{CT}$ or MR $[9,13]$. However, there was considerable variation in the diagnostic performance of CEUS among the different patterns of IHP, and this variation should be further discussed.

CEUS proved adequately accurate in the detection of IHP of the target tumors, with a sensitivity of $85.7 \%$ and specificity of $100 \%$. These results can be easily explained, if we consider that detailed imaging of the target tumors was the first priority of each CEUS study. With repeated examinations, the operator became familiar with the enhancement pattern of each target tumor, and any increase, or reappearance of enhancing intratumoral foci could be confidently diagnosed in most of the cases. These results are in line with previous studies and a metaanalysis, which have demonstrated a high efficacy of CEUS in the focused evaluation of response of target tumors treated by TACE. In this context, CEUS is comparable (and occasionally superior) to CT [23]; a high degree of concordance has also been demonstrated when CEUS is compared with MR [7]. In a study of Zheng et al [10], in which CEUS was used for the follow-up of HCC after RFA, the authors reported a significantly lower sensitivity of CEUS $(67.5 \%)$ in detecting progression of the target tumors. However, compared to our targets, they studied significantly smaller tumors and they often encountered unfavourable imaging conditions. Our two diagnostic failures were of clinical relevance; if classification of response had been based only on CEUS findings, the two respective patients would have been considered as "complete responses" (instead of "progressive disease") and no additional treatment would have been offered.

Despite its performance in the follow-up of target tumors, CEUS showed a very low sensitivity $(40 \%)$ in the diagnosis of IHP of non-target tumors. We acknowledge that this percentage is derived from a very small number of cases, but we believe that it is realistic and it is explained by the limited ability of CEUS for complete and simultaneous hemodynamic study of multiple lesions. Previous work $[19,24]$ has shown that a reliable evaluation of more than 3 treated tumors cannot be achieved with one or two injections of echo-enhancer. Additional injections would increase the duration and complexity of the study. Cost is also a concern, although very small doses $(1 \mathrm{ml})$ of the echo-enhancer are sufficient for high quality CEUS imaging with many modern machines [24]. Notably, IHP of non-target tumors (and consequently, its respective misdiagnosis by CEUS) is not always clinically significant. For example, a misdiagnosis on the growth of a small non-target tumor would have no clinical relevance for a patient with significant residual disease of his target tumors. Treatment continuation would be anyway required for this patient [7].

Our work also revealed a low sensitivity of CEUS in the detection of new intrahepatic recurrences. In the aforementioned study of Zheng et al, a sensitivity of $77.7 \%$ was observed, when CEUS was utilized for the detection of new liver lesions post RFA [10]. The inferior performance of CEUS in our study can be attributed to both technical and background factors: we performed only 2 injections per CEUS study (Zhang et al: 2-3 injections) and we scanned the liver for only 240 seconds 
(Zhang et al: $360 \mathrm{~s}$ ). Moreover, CEUS follow-up post TACE may be more challenging than CEUS post RFA, because patients treated with TACE usually have a higher number of target and non-target tumors than patients treated with ablation. Of note, only 1 case of new lesions (3.7\% of all cases of new lesions) was observed during the phase of the first 3 scheduled TACE sessions. There are also other studies, in which the appearance of new lesions was relatively unusual during the first months post treatment initiation, with a rate of $2.8 \%-4.8 \%[25,26]$. To estimate the clinical significance of the diagnostic failures of CEUS in the setting of new lesions, one should take into account the status of the preexisting target and non-target tumors, the extent and distribution of the new lesions and the patient's eligibility for further treatment. In a fit patient with complete response of the preexisting tumors, such a misdiagnosis would cause unacceptable delay in the onset of additional treatment.

The poor performance of sonography in the aforementioned setting reflects some inherent limitations of US and CEUS. On unenhanced US, difficulties in the detection of new HCC foci are caused by the isoechogenicity of some tumors and by the diffusely inhomogeneous and coarsened echotecture of the cirrhotic liver. On CEUS, scanning at the delayed phase may fail to detect wash-out of new lesions since some HCCs show a late and weak wash-out, or no wash-out at all [18]. Additional problems are caused by the heterogeneous parenchymal enhancement of the cirrhotic liver [27]. For these reasons, CEUS with vascular phase agents is not recommended for the detection of HCC in the cirrhotic liver [18].

In our work, CEUS proved efficient in the detection of IHP involving large liver veins. These vessels are routinely scanned during the baseline (unenhanced) post-interventional US study, and echogenic material within these veins can be easily depicted. If gray-scale and color Doppler cannot definitely diagnose the nature of venous thrombus, CEUS can be utilized to demonstrate neoplastic thrombus enhancement with high sensitivity and specificity [28].

According to some modern TACE protocols $[7,29,30]$, HCC patients are treated with at least 3 scheduled sessions of TACE at relatively short (4-7 weeks) intervals. As indicated by our results, IHP of new lesions and CEUS diagnostic failures are unusual during this period, while focused CEUS evaluation of the target tumors is reliable [7]. We hypothesize that, in selected patients (with limited number of lesions at baseline and suitable for high quality US imaging), CEUS could be used as the primary imaging modality after the first and second session of TACE. If significant residual tumor is diagnosed on these post-interventional CEUS studies, further treatment could be undertaken with no additional imaging.
Scanning with CT or MR could be reserved for the evaluation after the 3rd session of TACE.

There are several limitations of this study: there was a significant variation in the interval between the follow-up imaging studies, which probably reduces the accuracy in the calculation of the time of IHP. US machines of different technological background were utilized, and the effect of these differences on the diagnostic yield of CEUS is unknown. There was no histopathologic confirmation of the diagnosis of IHP, and we are aware that, despite its leading role in post-interventional imaging, MR has also limitations in the diagnosis of newly appearing lesions, with a reported sensitivity of only $44 \%$ [31].

\section{Conclusion}

This work provides additional evidence on the strengths and limitations of CEUS, when this modality is used to globally assess tumor-bearing liver post loco-regional treatment. Recognition of these limitations might result in a more appropriate and effective use of CEUS in the post-interventional setting. The role of CEUS as the primary imaging modality for short- to mid-term evaluation in selected TACE protocols probably warrants further research.

\section{Conflict of interest: none}

\section{References}

1. Chung YE, Kim KW. Contrast-enhanced ultrasonography: advance and current status in abdominal imaging. Ultrasonography 2015;34:3-18.

2. Roccarina D, Garcovich M, Ainora ME, et al. Usefulness of contrast enhanced ultrasound in monitoring therapeutic response after hepatocellular carcinoma treatment. World J Hepatol 2015;7:1866-1874.

3. Liu LN, Xu HX, Zhang YF, Xu JM. Hepatocellular carcinoma after ablation: the imaging follow-up scheme. World J Gastroenterol 2013;19:797-801.

4. Yamamoto K, Shiraki K, Nakanishi S, et al.1.5 Harmonic Imaging Sonography with microbubble contrast agent improves characterization of hepatocellular carcinoma. World J Gastroenterol 2005;11:5607-5613.

5. Pompili M, Riccardi L, Covino M, et al. Contrast-enhanced gray-scale harmonic ultrasound in the efficacy assessment of ablation treatments for hepatocellular carcinoma. Liver Int 2005;25:954-961.

6. Takizawa K, Numata K, Morimoto M, et al. Use of contrast-enhanced ultrasonography with a perflubutane-based contrast agent performed one day after transarterial chemoembolization for the early assessment of residual viable hepatocellular carcinoma. Eur J Radiol 2013;82:1471-1480. 
7. Moschouris H, Malagari K, Papadaki MG, et al. mRECIST criteria and contrast-enhanced US for the assessment of the response of hepatocellular carcinoma to transarterial chemoembolization. Diagn Interv Radiol 2014;20:136-142.

8. Liu M, Lin MX, Lu MD, et al. Comparison of contrast-enhanced ultrasound and contrast-enhanced computed tomography in evaluating the treatment response to transcatheter arterial chemoembolization of hepatocellular carcinoma using modified RECIST. Eur Radiol 2015;25:2502-2511.

9. Lencioni R, Llovet JM. Modified RECIST (mRECIST) assessment for hepatocellular carcinoma. Semin Liver Dis 2010;30:52-60.

10. Zheng SG, Xu HX, Lu MD, et al. Role of contrast-enhanced ultrasound in follow-up assessment after ablation for hepatocellular carcinoma. World J Gastroenterol 2013;19:855865.

11. Catalano O, Izzo F, Vallone $P$, et al. Integrating contrastenhanced sonography in the follow-up algorithm of hepatocellular carcinoma treated with radiofrequency ablation: single cancer center experience. Acta Radiol 2015;56:133142.

12. Rossi S, Ravetta V, Rosa L, et al. Repeated radiofrequency ablation for management of patients with cirrhosis with small hepatocellular carcinomas: a long-term cohort study. Hepatology 2011;53:136-147.

13. Malagari K, Pomoni M, Sotirchos VS, et al. Long term recurrence analysis post drug eluting bead (deb) chemoembolization for hepatocellular carcinoma (hcc). Hepatogastroenterology 2013;60:1413-1419.

14. Okuwaki Y, Nakazawa T, Shibuya A, et al. Intrahepatic distant recurrence after radiofrequency ablation for a single small hepatocellular carcinoma: risk factors and patterns. $\mathrm{J}$ Gastroenterol 2008;43:71-78.

15. Bruix J, Sherman M; Practice Guidelines Committee, American Association for the Study of Liver Diseases. Management of hepatocellular carcinoma. Hepatology 2005;42:1208-1236.

16. Moschouris H, Malagari K, Papadaki MG, Kornezos I, Matsaidonis D. Contrast-enhanced ultrasonography of hepatocellular carcinoma after chemoembolisation using drug-eluting beads: a pilot study focused on sustained tumor necrosis. Cardiovasc Intervent Radiol 2010;33:10221027.

17. Lencioni R, de Baere T, Burrel M, et al. Transcatheter treatment of hepatocellular carcinoma with Doxorubicin-loaded DC Bead (DEBDOX): technical recommendations. Cardiovasc Intervent Radiol 2012;35:980-985.

18. Claudon M, Dietrich CF, Choi BI, et al. Guidelines and good clinical practice recommendations for contrast enhanced ultrasound (CEUS) in the liver--update 2012: a WFUMBEFSUMB initiative in cooperation with representatives of AFSUMB, AIUM, ASUM, FLAUS and ICUS. Ultraschall Med 2013;34:11-29.
19. Moschouris H, Malagari K, Papadaki MG, et al. Short-term evaluation of liver tumors after transarterial chemoembolization: limitations and feasibility of contrast-enhanced ultrasonography. Abdom Imaging 2011;36:718-728.

20. Xu HX, Xie XY, Lu MD, et al. Contrast-enhanced sonography in the diagnosis of small hepatocellular carcinoma $<$ or $=2 \mathrm{~cm}$. J Clin Ultrasound 2008;36:257-266.

21. Kloeckner R, Otto G, Biesterfeld S, Oberholzer K, Dueber $\mathrm{C}$, Pitton MB. MDCT versus MRI assessment of tumor response after transarterial chemoembolization for the treatment of hepatocellular carcinoma. Cardiovasc Intervent Radiol 2010;33:532-540.

22. Lee YJ, Lee JM, Lee JS, et al. Hepatocellular carcinoma: diagnostic performance of multidetector CT and MR imaging-a systematic review and meta-analysis. Radiology 2015;275:97-109.

23. Tai CJ, Huang MT, Wu CH, et al. Contrast-Enhanced Ultrasound and Computed Tomography Assessment of Hepatocellular Carcinoma after Transcatheter Arterial ChemoEmbolization: A Systematic Review. J Gastrointestin Liver Dis 2016;25:499-507.

24. Sparchez Z, Mocan T, Radu P, Anton O, Bolog N. Contrast enhanced ultrasonography in assessing the treatment response to transarterial chemoembolization in patients with hepatocellular carcinoma. Med Ultrason 2016;18:96-102.

25. Malagari K, Pomoni M, Kelekis A, et al. Prospective randomized comparison of chemoembolization with doxorubicin-eluting beads and bland embolization with BeadBlock for hepatocellular carcinoma. Cardiovasc Intervent Radiol 2010;33:541-551.

26. Kinugasa H, Nouso K, Takeuchi Y, et al. Risk factors for recurrence after transarterial chemoembolization for early-stage hepatocellular carcinoma. J Gastroenterol 2012;47:421-426.

27. Wong GL, Xu HX, Xie XY. Detection of focal liver lesions in cirrhotic liver using contrast-enhanced ultrasound. World J Radiol 2009;1:25-36.

28. Tarantino L, Ambrosino P, Di Minno MN. Contrast-enhanced ultrasound in differentiating malignant from benign portal vein thrombosis in hepatocellular carcinoma. World J Gastroenterol 2015;21:9457-9460.

29. Malagari K, Pomoni M, Moschouris H, et al. Chemoembolization of hepatocellular carcinoma with HepaSphere 30-60 $\mu \mathrm{m}$. Safety and efficacy study. Cardiovasc Intervent Radiol 2014;37:165-175.

30. Malagari K, Pomoni M, Moschouris H, et al. Chemoembolization with doxorubicin-eluting beads for unresectable hepatocellular carcinoma: five-year survival analysis. Cardiovasc Intervent Radiol 2012;35:1119-1128.

31. Marin HL, Furth EE, Olthoff K, Shaked A, Soulen MC. Histopathologic outcome of neoadjuvant image-guided therapy of hepatocellular carcinoma. J Gastrointestin Liver Dis 2009; $18: 169-176$. 